\title{
How Are We Handling the Post-COVID Patients? The Dance of Uncertainties
}

\author{
Donato Lacedonia Giulia Scioscia Cosimo Carlo De Pace Antonio Laricchiuta \\ Pasquale Tondo Roberto Sabato Maria Pia Foschino Barbaro
}

Department of Medical and Surgical Sciences, Foggia University Hospital, University of Foggia, Foggia, Italy

Dear Editor,

The emergence of severe acute respiratory syndrome coronavirus 2 disease (COVID-19) has caught off guard and shattered the entire scientific and medical world system $[1,2]$. Similar to post-acute viral syndromes described in survivors of other virulent coronavirus epidemics, there are increasing reports of persistent and prolonged effects after acute COVID-19 [3, 4]. The post-acute COVID-19 syndrome (PACS) is a syndrome characterized by persistent symptoms and/or delayed or long-term complications beyond 4 weeks from the onset of symptoms (long COVID) $[5,6]$. PACS affects many organ systems like pulmonary/cardiovascular (fatigue, dyspnea, and chest pain), hematologic (thromboembolic events), dermatologic, neuropsychiatric, and endocrine and results in decline in quality of life.

We present the results of a survey we submitted to 83 pulmonologists (sharing a form on JotForm www.jotform.com) from all over Italy during the month of March 2021. The results were then analyzed with Prism (GraphPad software). In this survey, we asked these specialists how they manage PACS, and then we divided the answers on the basis of groups per the patients affected by PACS (group 1: $<100$ and group 2: $>100$ ) visited.

\section{karger@karger.com www.karger.com/res \\ Karger"}

\section{(C) 2021 The Author(s)}

Published by S. Karger AG, Basel

GOPEN ACCESS
The survey composed of 15 questions. The first question concerned the time of visit: after what time from the clinical recovery and negativization on nasopharyngeal swab the pulmonologist suggest to make the first followup: $50 \%$ of the specialists suggested to make a visit after 1 month, but when we divided by the number of patients visited, we could see that almost $75 \%$ of pulmonologists of group 2 suggested to schedule a visit after 3 months (shown in Fig. 1a). As for how much time after the first medical check is more appropriate to revisit the patient, most agreed to set a follow-up after 3 or 6 months based on the symptoms referred to (no difference rose between the 2 groups of specialists).

The second part of the survey was about the functional and radiological exams to include in the follow-ups. Spirometry and diffusion lung carbon monoxide were the most common answers (almost $80 \%$ of the specialists believe that these exams are indicated for each follow-up). About the request of CT, most specialists (75\%), regardless of the number of patients evaluated, believe to schedule the exam during the 3 months of the follow-up visit (shown in Fig. 1b). We then asked if it is necessary to repeat CT during follow-up: $50 \%$ of the specialists suggested to make a control after 6 months, while $12 \%$ consid-

Correspondence to:

Giulia Scioscia, giulia.scioscia@ unifg.it 




Fig. 1. The 4 focal points of the analysis of the survey.

ered it unnecessary during further checks, $20 \%$ suggested to perform a CT at 1 year, and the remaining $18 \%$ believe that it is useful to repeat CT every 3 months. Furthermore, $63 \%$ of pulmonologists suggested to require an echocardiogram if during the visit the patient reported the symptom of dyspnea, while $20 \%$ always required an echocardiogram during the follow-up regardless of the symptoms, and the remaining $17 \%$ do not recommend a routine echocardiogram.

We also focused on the utility of D-dimers during the follow-up. Forty-six percent of specialists do not prescribe a routine blood test analysis during the follow-up (73\% in group 2), while $36 \%$ always prescribe blood test analysis including D-dimers. Specialists believe opportunely to provide further investigations according to the elevated value of D-dimers, specifically if it is 3 times the range according to the $42 \%$ or if it is over the range (15\%), while $26 \%$ of the participants analyze the value by comparing it to previous exams. However, if the value is over the range, $15 \%$ of the specialists prescribe low-molecularweight heparin, 33\% suggest to repeat the test after some weeks, and $42 \%$ prescribe an angio-CT and/or a Doppler ultrasonography of the lower limbs (shown in Fig. 1c).

Finally, the last part was about the treatment prescribed for dyspnea, fatigue, or psychological issues. Thirty percent of the specialists in the evidence of dyspnea suggest physiokinesiotherapy and/or use of mechanical device (10\%), while almost $20 \%$ suggest oral corticosteroids (OCS) usually if there is still the presence of ground glass in the CT (in this case, the OCS are suggested for a month by $50 \%$ of the specialists and for a few days by $25 \%$ ). About $10 \%$ of the responses also include the use of ICS/LABA, regardless of the presence or absence of an obstructive deficit on spirometry. The remaining $34 \%$ of 
Table 1. Comparison between BTS guidelines and our real life

\begin{tabular}{|c|c|c|}
\hline Question & BTS guidelines & Real life \\
\hline $\begin{array}{l}\text { When to perform the first post-COVID } \\
\text { visit? }\end{array}$ & $\begin{array}{l}3 \text { months } \\
4-6 \text { weeks, telephone consultation or visit in } \\
\text { selected cases }\end{array}$ & $\begin{array}{l}\text { Usually after } 3 \text { months } \\
1 \text { month only in selected cases }\end{array}$ \\
\hline $\begin{array}{l}\text { How often to perform the post-COVID } \\
\text { follow-up after first visit? }\end{array}$ & $\begin{array}{l}\text { Never (no alteration at CXR or PFT) } \\
6-8 \text { weeks in case of persistent alteration at } \\
\text { CXR }\end{array}$ & $\begin{array}{l}\text { Usually after } 6 \text { months, every } 3 \text { months only } \\
\text { in selected cases }\end{array}$ \\
\hline Which lung function tests to perform? & Spirometry, DLCO, 6MWT in selected cases & Spirometry, DLCO, 6MWT in all subjects \\
\hline When to perform control CT? & Only if there is abnormal CXR or PVD & $\begin{array}{l}3 \text { months after negativization in } \\
\text { symptomatic patients }\end{array}$ \\
\hline $\begin{array}{l}\text { After how long to carry out the control } \\
\text { CT? }\end{array}$ & No further indications & Usually after 6 months \\
\hline $\begin{array}{l}\text { What to do for the treatment of } \\
\text { dyspnea? }\end{array}$ & $\begin{array}{l}\text { No specific therapy } \\
\text { Pulmonary rehabilitation }\end{array}$ & Physical activity, physiokinesis therapy \\
\hline When to use corticosteroids? & Not indicated & $\begin{array}{l}\text { Only in selected cases in which a pulmonary } \\
\text { or systemic inflammatory state is evident }\end{array}$ \\
\hline How to treat fatigue? & $\begin{array}{l}\text { No specific therapy } \\
\text { Rehabilitation }\end{array}$ & $\begin{array}{l}\text { Supplements } \\
\text { Physical activity }\end{array}$ \\
\hline Anxiety and depression & Consider psychological support & Consider psychological support \\
\hline
\end{tabular}

the responses included no therapy, mucolytics, supplements, or vitamins (shown in Fig. 1d). For the treatment of fatigue, $33 \%$ of participants prescribed regular physical activity, 25\% physiokinesiotherapy, and 30\% supplements or vitamins, and in most cases, a combination of them. With regard to the management of other clinical manifestations typically associated with COVID (psychological, neurological, and renal disorders), none of the interviewees works in a center with other specialists in a multidisciplinary team, and evaluations are requested only in the case of the presence of specific symptoms. Fifty percent of the specialists prescribe a psychological evaluation with an affiliated specialist in selected cases while $30 \%$ only suggest an evaluation, but the patient has to provide by himself to find a specialist.

The results of this survey show that there is still a lot of uncertainty about the management of PACS. In fact, in a few cases, an agreement of $>70 \%$ was reached. In general, there is good agreement on the timing of the follow-up and the type of functional, radiological, and serological analyses considered appropriate to perform, while there is much disagreement on the therapies to be recommended in case of persistence of dyspnea, asthenia, or high values of D-dimers.

In a study recently published in Nature (March 22, 2021) [7], it is recommended that clinical assessment and chest X-ray have to be guaranteed in all patients at 12 weeks while pulmonary functional tests (PFTs), 6-min walking tests (6MWTs), sputum sampling, and echocardiogram have to be provided according to clinical judgment; moreover, patients are recommended to be evaluated with chest high-resolution CT, CT pulmonary angiogram, or echocardiogram, or discharged from follow-up. In our analysis, the specialists prefer CT rather than chest X-ray, while they do not usually provide sputum sampling. In addition, an earlier clinical assessment for respiratory, psychiatric, and thromboembolic sequelae, as well as rehabilitation needs, is recommended at 4-6 weeks after hospital discharge for patients with severe acute COVID-19, defined as those who had severe pneumonia, required ICU care, are elderly, or have multiple comorbidities. According to our survey, there is a mis- 
match between groups because group 1 provides the first visit after 4 weeks and group 2 after 12 weeks. About the treatment with OCS, the authors suggest that it may be beneficial in a subset of patients with post-COVID inflammatory lung disease, according to a preliminary observation of significant symptomatic and radiological improvement in a small UK cohort of COVID-19 survivors. Studies are still ongoing, but, in our survey, almost $20 \%$ of specialists suggest OCS only if there is still ground glass in the CT, while physiokinesiotherapy is usually preferred.

While this study suggests the treatment with corticosteroids, it does not suggest for how long the patient has to take them. This is controversial also in our survey because we received many different opinions not only in the decision to administer the drug or not, but also in terms of posology and method of administration.

Waiting for further scientific works and official guidelines that give clear indications on what is the most appropriate management of PACS patients, based on the data emerging from this survey, we summarize in Table 1 the main indications emerged in clinical practice and compare it with suggestions of BTS guidelines [8]. Compared to what is suggested by the BTS guidelines, in our sample, there is a more frequent use of CT and a greater number of follow-up visits after the first one. Furthermore, the use of corticosteroids is frequent in real life, although there are no specific indications.

In conclusion, despite the rich literature on this topic, the possibility of having clear recommendations about the management of patients affected by PACS still seems far away, in terms of timing of visits, clinical and functional assessments, and treatment. According to our survey, there is a tacit common line of action diffused among the pulmonologists with only some little differences in the management according to the number of patients following up. Main open issues should be solved with standardized European guidelines in order to obtain a better clinical practice for optimizing the safety of patients and the administration of health-care resources.

\section{Acknowledgments}

We want to thank all the pulmonologists of the group PneumovsCovid and PneumologiaPuglia that participated in the survey and in these months shared information and experience in the fight against COVID.

\section{Conflict of Interest Statement}

The authors have no conflicts of interest to declare.

\section{Funding Sources}

No funding was provided to any authors of this study.

\section{Author Contributions}

All authors (Donato Lacedonia, Giulia Scioscia, Cosimo Carlo De Pace, Antonio Laricchiuta, Pasquale Tondo, Roberto Sabato, and Maria Pia Foschino Barbaro) contributed equally to the study.

\section{References}

1 Mohamadian M, Chiti H, Shoghli A, Biglari S, Parsamanesh N, Esmaeilzadeh A. COVID-19: virology, biology and novel laboratory diagnosis. J Gene Med. 2021;23(2).

2 Umakanthan S, Sahu P, Ranade A, Bukelo $\mathrm{MM}$, Rao JS, Kv D, et al. Origin, transmission, diagnosis and management of coronavirus disease 2019 (COVID-19). Postgrad Med J. 2020;96(1142):753-8.

3 Mendelson M, Nel J, Blumberg L, Madhi SA, Dryden M, Stevens W, et al. Long-COVID: an evolving problem with an extensive impact. $S$ Afr Med J. 2020;111(1):10-2.
4 el Sayed S, Shokry D, Gomaa SM. Post-COVID-19 fatigue and anhedonia: a cross-sectional study and their correlation to post-recovery period. Neuropsychopharmacol Rep. 2021; 41(1):50-5.

5 Moreno-Pérez O, Merino E, Leon-Ramirez JM, Andres M, Ramos JM, Arenas-Jiménez J, et al. Post-acute COVID-19 syndrome. Incidence and risk factors: a Mediterranean cohort study. J Infect. 2021;82(3):378-83.
6 Huang C, Huang L, Wang Y, Li X, Ren L, Gu $\mathrm{X}$, et al. 6-month consequences of COVID-19 in patients discharged from hospital: a cohort study. Lancet. 2021;397(10270):220-32.

7 Nalbandian A, Sehgal K, Gupta A, Madhavan M, McGroder C, Stevens IS, et al. Post-acute COVID-19 syndrome. Lancet. 2021;27(4): 601-15.

8 George PM, Barratt SL, Condliffe R, Desai SR, Devaraj A, Forrest I, et al. Respiratory followup of patients with COVID-19 pneumonia. Thorax. 2020;75(11):1009-16. 